Fecha de recepción: octubre 2021

Fecha de aprobación: noviembre 2021

Fecha publicación: diciembre 2021

\section{Identidad gráfica de Gucci y Fendi. El signo identificador y su rendimiento gráfico}

María Laura Spina ${ }^{(1)}$

Resumen: Es indispensable descubrir los parámetros de rendimiento de los signos gráficos cuando forman parte de un sistema gráfico. Si bien, la función de dichos parámetros es específicamente técnica, puede observarse un comportamiento comunicacional objetivo del signo identificador en el contexto real.

Es razonable cuestionar las expresiones propias del signo, vigencia, versatilidad, legibilidad, calidad gráfica genérica, aún en aplicaciones en micro escala. Formando parte de un sistema de identidad visual donde se aplican determinados recursos visuales en diferentes soportes, las marcas Gucci y Fendi demuestran, en algunas de sus aplicaciones, una ausencia de condicionamientos a partir del uso de parámetros independientes, permitiendo alcanzar un correcto comportamiento de la identidad.

Palabras clave: Signo - identidad - soporte - escala - microescala - trama visual - moda lujo - Gucci - Fendi - sistema gráfico.

[Resúmenes en inglés y portugués en las páginas 44-45]

(1) Diseñadora Gráfica Universidad de Buenos Aires. Profesora de la Universidad de Palermo en el Departamento de Diseño Visual y Comunicación Corporativa y Empresaria de la Facultad de Diseño y Comunicación.

\title{
Introducción
}

En el ámbito de la moda, las firmas italianas Gucci y Fendi se destacan por la fabricación de artículos de lujo y por la constante aplicación de sus signos marcarios en cada uno de sus productos. En el contexto en el que estas firmas se desempeñan, sus signos identificadores actúan con una función específica, individualizando a cada entidad.

Se entiende por identificador corporativo a aquel signo visual de diferentes tipologías, tales como monograma, logotipo, símbolo, mascota.

Dentro de este identificador visual se encuentra el logotipo, de naturaleza verbal, con una función específicamente denominativa. En ocasiones, es acompañado por un identificador no verbal de naturaleza icónica o simbólica.

Cada uno de ellos se rodea de rasgos descriptivos o valorativos ampliando su significado y reforzando el posicionamiento social de la entidad. 
Según Costa (2005), el repertorio de signos se re-codifica en dos categorías: las marcas icónicas o figurativas con sus componentes gráfico y funcional y el logotipo o marca verbal cuyo origen proviene del enlace de letras provenientes de la imprenta, las artes gráficas o la fundición de tipos de imprenta. Estas últimas son marcas para ser leídas, mientras que las primeras son para ser vistas.

Tanto Gucci como Fendi son marcas para ser vistas y leídas, pues buscan una constante interacción con sus públicos a través de los atributos que se van asignando a cada uno de sus identificadores visuales, cumpliendo cada una con la función de marcaje de la identidad. Ambas firmas poseen un capital marcario individual que consta de la acumulación de valores asignados a la organización aplicados en su marca gráfica.

Estas dos empresas italianas son representadas por marcas gráficas, las cuales acompañan con alto rendimiento la aplicación de las mismas en cada una de las piezas visuales de comunicación.

$\mathrm{Al}$ ser marcas diseñadas para durar en el tiempo, tienen más posibilidades de ser aplicadas por ser estables, sólidas y equilibradas. En simultáneo, al ser marcas correctamente gestionadas en su aplicación y programación integral, que buscan equilibrar el aspecto técnico y visual, son capaces de desarrollar más probabilidades de posicionamiento estratégico, dado que esta comunicación al público por diferentes medios completará de contenidos y significados a cada una de las marcas.

Tanto Gucci como Fendi son marcas cuyos signos visuales son pertinentes estilísticamente al perfil de cada organización y actúan sin contradecir los valores o atributos a comunicar de cada empresa. Estas marcas, en un primer nivel comunicativo, se asocian directamente con las firmas Gucci y Fendi, obteniendo el grado de recordación que se necesita para que cada una cumpla con sus funciones básicas. En un segundo nivel de comunicación, ambas marcas se presentan como partes inseparables de las entidades a las que representan, se vuelven institucionalizadas, requiriendo el cumplimiento de todos los indicadores de calidad, comunicación y difusión.

De este modo, las marcas Gucci y Fendi son las únicas posibles para referir a sus entidades, consagrándose a ellas y volviéndose autónomas, adquiriendo independencia y valor propio. Para que estos niveles de comunicación sean eficaces, deben complementarse factores diversos: la calidad gráfica del signo identificador, la constancia y correcta gestión en sus aplicaciones de uso y los valores que se quieren comunicar en el contexto adecuado. Aún actuando en innumerables aplicaciones en microescala, Gucci y Fendi satisfacen las exigencias prácticas de la identificación siendo pertinentes al perfil de sus organizaciones manteniendo sus valores marcarios.

\section{Parámetros de alto rendimiento aplicados a Gucci y Fendi}

Los parámetros de alto rendimiento tienen una función meramente técnica que, con rigor analítico y objetividad, permiten una observación meticulosa de los signos de identidad en un contexto real. Tal como expresan Norberto Chaves y Raúl Belluccia: 
Se considera "gráfica corporativa de alto rendimiento" precisamente a aquella que da respuesta satisfactoria y completa a esas condiciones de identificación objetivas y particulares de cada caso concreto (Chaves y Belluccia, 2003, p. 39).

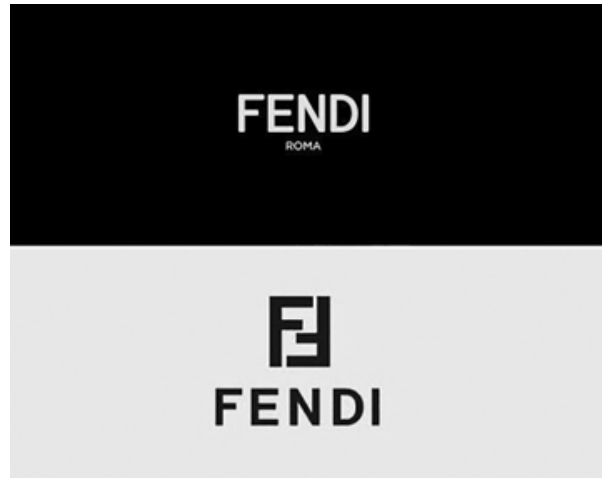

1

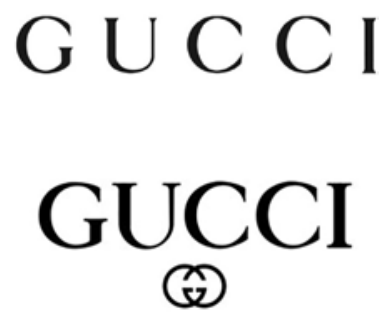

2

Figura 1. Identidad gráfica de Fendi. Fuente: https://www.logolynx.com/topic/fendi+roma. Figura 2. Identidad gráfica de Gucci. Fuente:https://www.logolynx.com/topic/gucci+official\#\&gid=1\&pid=6

Existen catorce parámetros de alto rendimiento aplicables al análisis y comportamiento de las marcas Gucci y Fendi.

Calidad gráfica genérica: permite comparar la calidad gráfica de dos marcas corporativas, determinar su equivalencia o la superioridad de una sobre la otra. Aquí, los elementos que componen un identificador son sujeto de análisis, destacando tanto la calidad de la familia tipográfica, como del diseño, de los elementos que lo componen, su cromaticidad y la aplicación de recursos visuales.

El análisis de este parámetro logra identificar que tanto Gucci como Fendi son marcas reconocibles, inequívocas y representativas en su sector.

Este indicador alude a la calidad de las organizaciones detrás de cada una de las marcas que las representan.

Ajuste tipológico: permite determinar la variedad de signos a utilizar según cada necesidad identificatoria concreta. Cada necesidad definirá qué tipo de signos son obligatorios y cuáles se desaconsejan. Teniendo presente que cada tipo de signo tiene sus cualidades, 
posibilidades y limitaciones, que determinan su adecuación o inadecuación en cada pieza de comunicación brindando confianza al consumidor, se observa que las marcas Gucci y Fendi, con sus características gráficas, responden con claridad a este parámetro.

Corrección estilística: este indicador logra inscribir al signo identificador en el contexto referencial de la organización describiendo su personalidad. El estilo condiciona los contenidos semánticos. Esta interpretación del mensaje visual no es sólo detectar el contenido semántico de cada marca sino su propio estilo, el que le da una característica propia a cada empresa. De este modo, las marcas Gucci y Fendi representan, a través de su estilo gráfico, el concepto de cada una de las empresas.

Compatibilidad semántica: este parámetro se verifica en la correcta ubicación de los signos. Los mismos, no deben hacer referencia a significados incompatibles o contradictorios con la identidad de la organización. No se deben forzar referencias a ciertos rasgos de la misma. Esto explica la síntesis y austeridad de elementos visuales en las marcas Gucci y Fendi donde sus componentes actúan con correcta compatibilidad semántica en ambos casos.

Suficiencia: este parámetro indica el grado de satisfacción de las necesidades de identificación mediante los signos disponibles. Los mismos son suficientes cuando alcanzan para cubrir las necesidades, pero, además, cuando no sobran. Cada elemento que compone visualmente las marcas Gucci y Fendi son importantes y suficientes.

Versatilidad: los identificadores deben concebirse para ser compatibles con todos los discursos. No es necesario que se adapten a la fuerza con variables secundarias, sino, por el contrario, deben concebirse inicialmente para formar parte de un sistema. Los signos de identidad gráfica de las entidades Gucci y Fendi atienden a un rendimiento parejo en todos los niveles de discurso sin perder su uniformidad, dado que cada mensaje emitido por las empresas mencionadas, confirma la existencia monolítica de la marca. Ninguna de las apariciones de marca evidencian incompatibilidad con el discurso.

Vigencia: este parámetro indica el tiempo de vida del signo identificador, el cual depende de la vida útil de los lenguajes visuales con que se construyó. Los lenguajes pueden pertenecer a una época y caducar con ella aunque hay lenguajes que continúan siendo actuales a pesar de los cambios culturales y sociológicos. Gucci y Fendi poseen lenguajes visuales en permanente actualidad, vigentes en el tiempo, sin hacer perder validez ni jerarquía a las empresas a las que identifican.

Reproducibilidad: la verificación, a través de este parámetro, se vincula con el tipo de soporte material pertinente, el cual plantea sus propias condiciones de reproducción. Este indicador plantea el desafío físico de los identificadores visuales dado que la reproducción en diversos soportes y situaciones, tales como pantallas de celular, computadoras y televisión, papel, alto y bajo relieve, volumen, transiluminados, crean diversas exigencias que condicionan directamente la forma del identificador visual. Gucci y Fendi son identificadores visuales que han sido diseñados para ser reproducidos correctamente en el 
repertorio completo de aplicaciones. De todos modos, con las nuevas aplicaciones tecnológicas pueden surgir algunas situaciones imprevistas que puedan presentar algún grado de imperfección con respecto a la versión original de marca.

Legibilidad: Este criterio está íntimamente vinculado con el anterior. Indica el grado de reconocibilidad visual de los rasgos esenciales del signo. Es un parámetro perceptual. Sus valores óptimos dependen de las condiciones particulares de lectura a las que esté sometido el signo identificador. Se busca una potente claridad de lectura, incluso por cromia, ya que el color corporativo se visualiza aún antes que la forma gráfica. En tal sentido, debe tenerse en cuenta la velocidad de lectura, donde la rápida detección del signo en un contexto visual urbano de alta complejidad, no solo exige una lectura en movimiento, ya sea vehicular o peatonal, sino también con condiciones de aceleración psíquica impuestas por el ritmo urbano que, en varias oportunidades, vuelve inaceptable el esfuerzo de la lectura. Las marcas correspondientes a Gucci y Fendi aún en publicidad urbana logran una correcta legibilidad favoreciendo la presencia marcaria de las mismas.

Inteligibilidad: esta capacidad del signo para ser comprendido en condiciones normales de lectura, es un parámetro que se aplica no solamente a formas abstractas sino también figurativas. Este criterio busca que el público vea directamente y del modo más rápido y claro posible lo que la marca pretende comunicar a través de su morfología. Si esto no sucediera, el signo se vuelve frágil y deficiente. Si bien, en la estrategia de identificación, los signos pueden ser abiertos o cerrados, tanto en lo icónico como verbal, el significado puede volverse explícito o no. Los signos correspondientes a Gucci y Fendi poseen un tipo de interpretación dominante que coincide con la intención identificadora adecuada logrando que sus marcas se reconozcan rápidamente. Es muy perjudicial que en una forma abstracta se vea algo real del mismo modo que en una forma figurativa, lo que se pretende comunicar, no se pueda detectar.

Pregnancia: este indicador es de gran importancia dado que refiere a la capacidad que tiene una forma para ser recordada. Implica la memoria del lector o también llamada memoria gráfica. Tanto Gucci como Fendi evidencian una lógica compositiva que no sólo facilita su registro sino también su retención óptica, la cual permite su recuerdo constante y su posterior reconocimiento.

Vocatividad: atraer la mirada es el principal objetivo de la vocatividad. Es la capacidad del signo de llamar la atención, imponiéndose con fuerza propia. En algunos casos, la vocatividad puede expresarse a través del color de alto impacto, o la fuerza morfológica, o el protagonismo de las formas por proporción o tamaño, o el grado de expresividad de los signos que componen la marca. Gucci y Fendi son dos marcas que acentúan su vocatividad a través de los elementos tipográficos que las componen y del equilibrio visual de las formas compositivas.

Singularidad: esta acepción busca hacer distinguir a la marca de las demás, asignando algún elemento que individualiza al sujeto. Las marcas correspondientes a Gucci y Fendi son 
singulares tal como lo exige la propia identidad. Se diferencian entre sí, se reconocen como competencia, pero, en simultáneo, cada una mantiene un grado propio de singularidad. La singularidad de un signo llega a poseer su máxima potencia cuando se ajusta al grado requerido para cada caso.

Declinabilidad: este último parámetro implica que el signo gráfico se reconozca en un código y pueda ser reproducido y seriado. Se aplica para hacer cumplir el grado de codificabilidad que se requiere. Es posible, para lograrlo, construir familias de marcas donde los propios signos básicos puedan descomponerse para crear nuevos signos similares entre sí pero reconocibles como miembros de un todo visual.

El universo marcario de Gucci y Fendi mantiene este criterio ya que, en ambos casos, los elementos tipográficos y las gamas cromáticas que se aplican regularmente en las diversas aplicaciones de marca facilitan la codificación de mensajes nuevos incluyéndose dentro del discurso de cada una de las organizaciones y aludiendo constantemente a sus identidades.

\section{El concepto de sistema gráfico asociado a la marca}

Un sistema gráfico determina qué elementos o recursos visuales son necesarios aplicar para reforzar el concepto de marca. Para ello, de acuerdo al grado de simplicidad o complejidad del sistema, se recurre o apela al uso de la microescala o de la macroescala aplicadas en distintos soportes visuales.

Sin embargo, las características de estos sistemas se determinan, por otro lado, según la cantidad de imágenes a utilizar, las variables y fuentes tipográficas, la diversidad de superficies de aplicación, las relaciones entre los elementos y las relaciones de estos elementos en el campo donde se aplican.

Realizar un sistema gráfico general involucra procesos analíticos, decisionales y propositivos con el fin de lograr sistemas particulares dando lugar a un sistema de sistemas.

Aquellos sistemas cerrados pueden entenderse como simples o de baja complejidad debido a la poca diversidad de elementos gráficos utilizados, a las variables limitadas, a las pocas alternativas de aplicación junto a un repertorio acotado de tipografías e imágenes y una propuesta cromática limitada. Este tipo de sistemas autosuficientes o cerrados funcionan con reglas y datos predefinidos.

Por otro lado, los sistemas abiertos o complejos implican una mayor diversidad de elementos: imágenes, tipografías, cromas, superficies de aplicación, un fundamento conceptual relevante y versatilidad en las aplicaciones, tales como diversas tipologías, contextos de intervención y variables de escala. Además, permiten la influencia de una fuente externa: color, forma, imagen, tipografía, ilustraciones.

Ambos tipos de sistemas, cerrados o simples y abiertos o complejos deben ser ricos en cuanto a la definición de sus elementos y flexibles, en cuanto a la utilización de constantes y variables visuales. Los atributos de los elementos del lenguaje visual y la cromia junto con la articulación de los mismos en el campo visual pueden variar a lo largo del sistema. Por lo tanto, es de suma importancia definir cuánto se va a mantener constante y cuánto 
va a variar en cada una de las composiciones que deberán resolverse. Para ello, es necesario establecer un criterio visual, el cual es producto del proceso de construcción del sistema gráfico.

Reforzando esta tipología, surge el concepto de sistemas fluidos, donde una identidad visual flexible abandona la idea de signo identificador como pieza central de la identidad visual, dedicándose al desarrollo de sistemas visuales que controlen dichas identidades.

Este tipo de identidades dinámicas, también son conocidas como identidades líquidas o identidades fluidas por ser generativas, sensibles, vivas y en permanente evolución.

Estas identidades flexibles surgen como una contrapuesta a las identidades visuales estáticas, basadas en un diseño de logotipo fijo.

La idea básica del esquema es que una identidad visual flexible debe tener un equilibrio entre sus constantes y sus variables, que permiten que la identidad visual se adapte a las demandas permanentes de comunicación, siendo cada uno de sus componentes flexible o estático. La flexibilidad también puede venir de la aplicación del sistema visual, puede adaptarse a los medios de comunicación, a la forma y también a los contenidos.

En un sistema visual estático, por lo general, se aumenta o reduce el tamaño de marca. No se adapta flexiblemente, no puede adaptar su forma y menos, su contenido.

Gucci y Fendi trabajan y actúan como sistemas semi abiertos pero al mismo tiempo flexibles, donde la constante es la aplicación permanente del signo identificador en escala reducida o microescala generando, en algunas aplicaciones, tramas visuales con la marca como módulo de repetición. Para lograr las variables, ambas marcas ponen en crisis lo que es posible variar para mantener el sentido de unidad y generan aplicaciones con tramas en movimiento y modificaciones cromáticas. Ambas marcas buscan en sus sistemas gráficos un equilibrio visual y apertura a nuevas transformaciones para no desvirtuar la unidad del sistema total y mantener en sus propuestas de identidad toda su potencia expresiva.

\section{El concepto de escala asociado a la marca}

La escala es la capacidad o propiedad que tienen los elementos visuales para relacionarse, modificarse o repercutir unos a otros. La distancia de observación modifica o altera las formas pero las relaciones entre las formas, los objetos y su periferia pueden controlarse mediante un uso correcto de la escala.

Para llevar a cabo este procedimiento, fue ideado el metro patrón cuya medida es el hombre. El reconocido arquitecto Le Corbusier creó un canon proyectual basado en medidas humanas, denominado Modulor. El mismo trata de dimensionar y contornear las medidas del ser humano al hallarse éste de pie, sentado o acostado y, de acuerdo a estas dimensiones, se creó una espiral matemática apta para establecer múltiples relaciones entre el hombre y la arquitectura. Este riquísimo patrón ergonómico permite concebir las cosas a escala y medida del hombre.

Según afirma Le Corbusier: 
El Modulor no concede talento y, mucho menos, genio. No convierte en sutil lo espeso. Les ofrece la facilidad que puede resultar del empleo de medidas seguras, pero son ustedes quienes tienen que elegir en el ilimitado depósito de las combinaciones del Modulor (Le Corbusier, 1953, p. 122).

En esta línea de pensamiento, González Ruiz (1994) plantea que se deben controlar las relaciones de las cosas entre sí y entre ellas y el hombre, pues el mismo hombre es quien establece el canon visual, por el cual las cosas son grandes o pequeñas, anchas o estrechas, imponentes o mínimas tanto entre sí como en relación al ser humano.

Por lo tanto, el concepto de escala es aplicable a las marcas Gucci y Fendi. En ambas se adjudica equilibrio a cada una de las partes que las componen $y$, cuando necesitan aplicarse en diferentes espacios o soportes, mantienen la proporción y la escala correspondientes.

Si bien cada superficie de aplicación como papelería corporativa, indumentaria, web, redes sociales, packaging, publicidad, editorial, poseen características propias, un uso correcto de la escala en ambas marcas logra mantener en equilibrio la forma y la función propiamente dicha de cada pieza gráfica donde la marca es aplicada.

Los criterios de organización y escala de marca están en relación con el formato y escala de cada una de las superficies de aplicación. Los formatos con los que se trabaja tienen estructuras distintas, por lo tanto, para poder apropiarse del campo, es necesario reconocer esas estructuras y establecer un correcto diálogo visual con ellas.

\section{La microescala aplicada visualmente}

Así como en innumerables proyectos de arquitectura y diseño se trabaja con el concepto de arquigrafía, es decir, gráfica aplicada a la arquitectura con un aumento ampliado de la propuesta gráfica respecto al tamaño del hombre en su macroescala, en los productos de lujo de las empresas Gucci y Fendi, se observa la aplicación de la marca gráfica en sentido contrario, conocido como microescala.

Los diferentes artículos de diseño de ambas marcas, poseen una propuesta gráfica de marca aplicada en un sentido reducido respecto al tamaño del ser humano sin perder la marca su capacidad de comunicación.

En este sentido Costa sostiene que "la identidad es el ADN de las empresas" (2003, p. 85). Según este autor, la imagen corporativa no se da solamente en función de lo que el diseño y sus formas gráficas transmiten. Lo que constituye la imagen pública de la empresa se constituye por la conducta de la misma, con su cultura, sus servicios, sus productos, su calidad, su capacidad para la innovación, su capacidad para vincularse con su público. Para ello, es necesario crear símbolos significativos y duraderos que al actuar con los elementos que los componen en total integración, constituyan los signos de identidad de las empresas.

A través de la aplicación de estos signos en escala reducida o microescala en diversos productos de cada una de las empresas mencionadas, se mantiene vigente la identidad de las mismas. Estas aplicaciones hacen visible, presente, memorizable y perceptible la identi- 
dad propia de Gucci y Fendi. En algunos productos, la aplicación de microescala se da exclusivamente en el signo identificatorio, mientras que en otros, se propone una trama visual utilizando el mismo signo gráfico como módulo de repetición. En ambos casos, la identidad se mantiene y se refuerza a través de estas propuestas. Tal como explica Costa:

Esta es la auténtica clave: ¿qué representa una marca de identidad? La pregunta no se interesa por lo que "es" objetivamente una marca, sino por lo que significa para el público. Una marca vale lo que representa (Costa, 2003, p. 89).

\section{La microescala y sus combinaciones}

La aplicación del lenguaje visual permite la creación del diseño, donde confluyen conceptos, formas, reglas y principios de organización visual. Debido a que los elementos conceptuales no son visibles, surgen en contraposición, el punto, la línea o el plano, como formas visibles y reconocibles.

Por lo tanto, las formas son reconocibles en toda superficie bidimensional, donde pueden colocarse con cambios cromáticos, interrelacionadas entre sí, en volumen, en positivo y negativo o con efectos especiales. Una de las características de la forma es que puede variar su escala, aumentar de tamaño para ser aplicada en macroescala o reducir el mismo y producir la microescala.

En innumerables productos de lujo, Fendi aplica el signo identificador en micro - escala asociada al concepto de gradación.

La gradación es una experiencia visual donde los elementos visuales que se encuentran cerca del espectador parecen grandes y lejanos parecen pequeños.

En una estructura de repetición de formas unitarias o módulos, los mismos, al estar solos o combinados, producen diferentes efectos. Este concepto supone que se pueden desarrollar gradaciones de tamaño, forma, color, textura, dirección, posición, espacio y gravedad. En el plano visual de los objetos de Fendi, se puede observar que la relación entre los módulos y el plano de la imagen permanece constante, sin embargo, se verifica una progresión en el plano, indicando un cambio gradual de posición de los módulos dentro de las subdivisiones estructurales que conforman al diseño. Estos módulos ascienden o descienden, trasladándose en el espacio en una secuencia de movimientos regulares y graduales. A través de tramas en micro - escala del signo identificador con acompañamiento del efecto de gradación, se observa una ilusión óptica como resultado de este proceso, donde se percibe la propia velocidad de gradación. En los productos Fendi, la velocidad de gradación es lenta, creando un efecto de ilusión óptica relajado, donde el módulo en estado de constante repetición, se va modificando gradualmente sin generar tantas alteraciones. Según Wong:

La serie de gradación queda marcada por una situación inicial y una situación final. La cantidad de pasos entre las situaciones inicial y final determina tanto la velocidad como el alcance de la gradación (Wong, 1979, p. 43). 
Existen algunos modelos de gradación, entre ellos, el movimiento zigzag, el cual supone que los módulos de un mismo paso se disponen ubicándose en forma de zigzag, transformándose a una misma velocidad. Esta estructura de gradación aplicada en Fendi es similar a una de repetición, excepto que el módulo o estructura repetitiva cambia de tamaño ubicándose según una secuencia gradual y sistemática. Se percibe un cambio de tamaño y proporción en sus variaciones de expansión y condensación, con un cambio en la dirección que, junto a una mayor curvatura, generan una combinación morfológica de la forma volviéndola dinámica, flexible y atractiva visualmente. (Figuras 3 y 4)

A diferencia de Fendi, los productos de lujo Gucci portan como aplicación dentro del sistema gráfico la micro escala asociada al concepto de estructura de repetición.

En esta estructura, las formas unitarias también denominadas módulos, se ubican regularmente en un espacio dado, dando origen a una estructura formal, visible y ubicada en toda la superficie del diseño. Esta estructura es más simple y muy útil para cubrir grandes superficies.

Gucci aplica los módulos de repetición, con el signo identificador en microescala en la mayor parte de la superficie de sus productos. A partir de una estructura invisible o inactiva, los módulos se ubican en las intersecciones de las líneas estructurales que, en su mayoría son ejes diagonales. Los módulos no se tocan entre sí y logran una correcta legibilidad en su ubicación en el espacio. (Figuras 5 y 6 )

\section{Conclusiones}

El comportamiento de los signos identificadores gráficos de Gucci y Fendi permite analizar un uso correcto de los mismos dentro de sus propios sistemas visuales, aún cuando actúan en escala reducida o micro-escala.

Los parámetros de rendimiento de marca dan cuenta de aplicaciones de marca correctamente gestionadas e integradas en un sistema de identidad visual versátil, sin dejar de tener en cuenta la funcionalidad de las mismas y la potencia expresiva de ambas marcas. Tanto Gucci como Fendi, logran proyectar una imagen corporativa sólida en el tiempo y eficaz teniendo presente la trayectoria de ambas empresas.

Sin perder de vista el contexto actual, la percepción y el contacto directo entre los productos, la empresa, la marca y los servicios, es posible afirmar que la imagen actual de ambas empresas es acorde a la cultura de las mismas, concreta en hechos, mensajes, conducta y comunicación. 


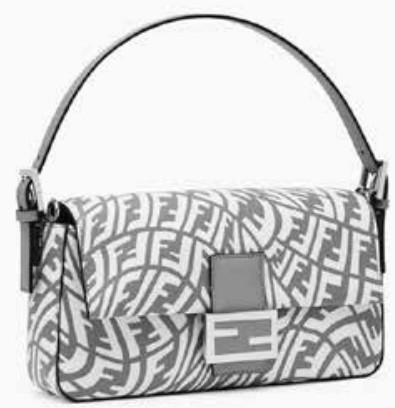

3

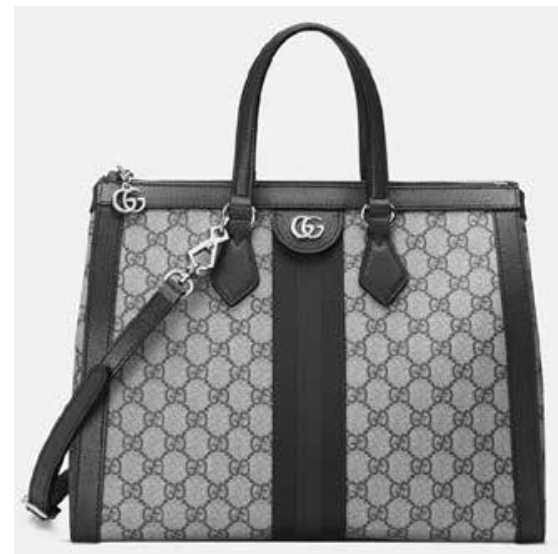

5

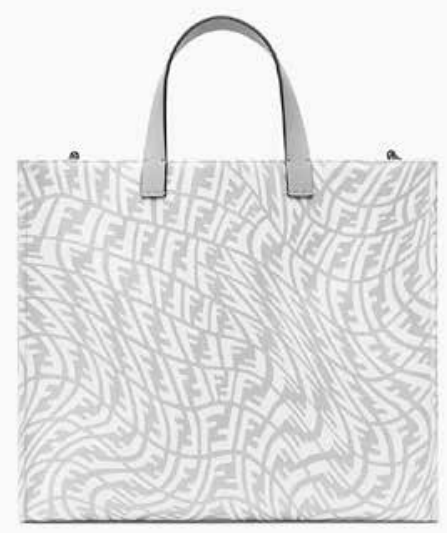

4

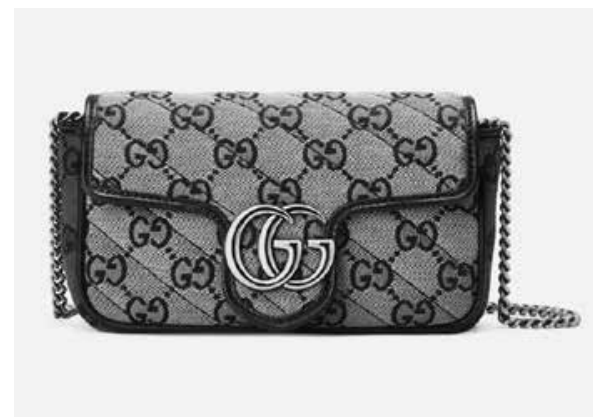

6

Figura 3. Bolso de dama Fendi con trama aplicada en movimiento en microescala y gradación. Fuente: https://www.fendi.com/it/donna/in-evidenza/summer-vertigo-woma. Figura 4. Tote bag de dama Fendi con trama aplicada en movimiento en microescala y gradación. Fuente: https://www.fendi.com/ it/donna/in-evidenza/summer-vertigo-woman. Figura 5. Tote bag de dama Gucci con trama aplicada en repetición y marca en microescala. Fuente: https:/www.gucci.com/au/en_au/pr/women/handbags/totebags-for-women/gucci-diana-medium-tote-bag-p-65565817QDT4972. Figura 6. Mini bag de dama Gucci con trama aplicada en repetición y marca en microescala. Fuente: https://www.gucci.com/au/en_au/ca/ women/handbags/mini-bags-for-women-c-women-handbags-mini-bags 


\title{
Lista de Referencias Bibliográficas
}

Arnheim, R. (2017). Arte y percepción visual. Barcelona: Alianza Editorial.

Chaves, N. (1990). La imagen corporativa. Barcelona: G. Gili.

Chaves, N; Belluccia, R. (2003). La marca corporativa: gestión de símbolos y logotipos. Buenos Aires: Paidós.

Costa, J. (2005). Imagen corporativa en el siglo XXI. Buenos Aires: La Crujía.

Costa, J. (1989). Imagen Global: evolución del diseño de identidad. Barcelona: CEAC.

Costa, J. (2003). Diseñar para los ojos. Bolivia: Grupo Editorial Design.

Frutiger, A. (1981). Signos, símbolos, marcas, señales. Barcelona: Gili.

González Ruiz, G. (1994). Estudio de diseño sobre la construcción de las ideas y su aplicación a la realidad. Buenos Aires: Emecé.

Le Corbusier, CH. (1953). El modulor. Ensayo sobre una medida armónica a la escala humana aplicable universalmente a la arquitectura y la mecánica. Buenos Aires: Editorial Poseidón. Wucius, W. (1979). Fundamentos del diseño bi- y tridimensional. Barcelona: G. Gili.

\begin{abstract}
It is essential to discover the performance parameters of graphic signs when they are part of a graphic system. Although the function of these parameters is specifically technical, an objective communicational behavior of the identifying sign can be observed in the real context.

It is reasonable to question the expressions of the sign, validity, versatility, legibility, generic graphic quality, even in micro-scale applications. As part of a visual identity system where visual resources are applied on different media, Gucci and Fendi brands show, in some of their applications, an absence of conditioning based on the use of independent parameters, allowing to achieve a correct identity behavior.
\end{abstract}

Keywords: Sign - identity - support - scale - microscale - visual fabric - fashion - luxury Gucci - Fendi - graphic system.

Resumo: É essencial descobrir os parâmetros de desempenho dos sinais gráficos quando eles fazem parte de um sistema gráfico. Embora a função desses parâmetros seja especificamente técnica, um comportamento comunicacional objetivo do signo de identificação pode ser observado no contexto real.

É razoável questionar as expressões do sinal, validade, versatilidade, legibilidade, qualidade gráfica genérica, mesmo em aplicações em microescala. Como parte de um sistema de identidade visual onde os recursos visuais são aplicados em diferentes mídias, as marcas Gucci e Fendi apresentam, em algumas de suas aplicações, uma ausência de condicionamento baseado no uso de parâmetros independentes, permitindo alcançar um comportamento de identidade correto. 
Palavras chave: Signo - identidade - suporte - escala - microescala - tecido visual - moda - luxo - Gucci - Fendi - sistema gráfico.

[Las traducciones de los abstracts fueron supervisadas por el autor de cada artículo] 